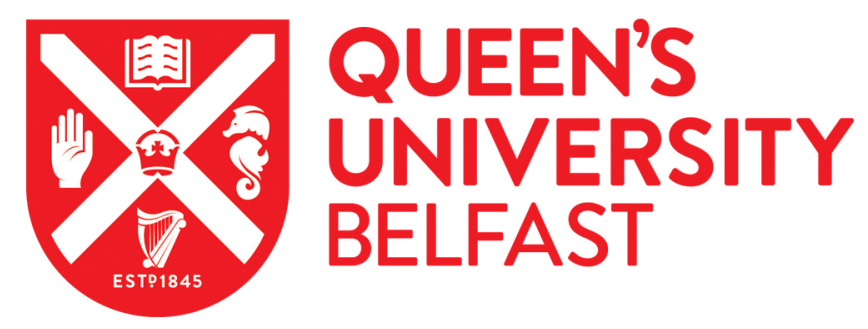

\title{
Effects of the availability of accurate proprioceptive information on older adults' postural sway and muscle co-contraction
}

\author{
Craig, C. E., Calvert, G. H. M., \& Doumas, M. (2017). Effects of the availability of accurate proprioceptive \\ information on older adults' postural sway and muscle co-contraction. European Journal of Neuroscience, \\ 46(10), 2548-2556. https://doi.org/10.1111/ejn.13703
}

Published in:
European Journal of Neuroscience

Document Version:

Peer reviewed version

Queen's University Belfast - Research Portal:

Link to publication record in Queen's University Belfast Research Portal

Publisher rights

Copyright 2017 Wiley This work is made available online in accordance with the publisher's policies. Please refer to any applicable terms of use of the publisher.

\section{General rights}

Copyright for the publications made accessible via the Queen's University Belfast Research Portal is retained by the author(s) and / or other copyright owners and it is a condition of accessing these publications that users recognise and abide by the legal requirements associated with these rights.

Take down policy

The Research Portal is Queen's institutional repository that provides access to Queen's research output. Every effort has been made to ensure that content in the Research Portal does not infringe any person's rights, or applicable UK laws. If you discover content in the Research Portal that you believe breaches copyright or violates any law, please contact openaccess@qub.ac.uk. 
DR. CHESNEY ELIZABETH CRAIG (Orcid ID : 0000-0002-9492-1294)

Article type : Research Report

Reviewers: Vassilia Hatzitaki, Aristotle University of Thessaloniki, Greece

Ramesh Balasubramaniam, University of California-Merced, USA

\section{Effects of the availability of accurate proprioceptive information on older adults' postural sway and muscle co-contraction}

Chesney E. Craig ${ }^{\mathrm{a}, \mathrm{b}}$, Glenn H. M. Calvert ${ }^{\mathrm{a}, \mathrm{c}}$, \& Michail Doumas $^{\mathrm{a}}$

\footnotetext{
${ }^{a}$ School of Psychology, Queen's University Belfast, Belfast, Co. Antrim, UK

${ }^{\mathrm{b}}$ Research Centre for Health, Exercise and Active Living, Department of Exercise and Sport Science,

Manchester Metropolitan University, Crewe, Cheshire, UK

${ }^{\mathrm{c}}$ Trinity College Institute of Neuroscience and School of Psychology, Trinity College Dublin, Dublin, IE

Corresponding author: Chesney Craig

Research Centre for Health, Exercise and Active Living, Department of Exercise and Sport Science, Manchester Metropolitan University, Crewe, Cheshire, United Kingdom

E: c.craig@mmu.ac.uk
}

RUNNING HEAD: Muscle co-contraction in older adults' posture

Key words: ageing; postural control; sensory integration; proprioception

This article has been accepted for publication and undergone full peer review but has not been through the copyediting, typesetting, pagination and proofreading process, which may lead to differences between this version and the Version of Record. Please cite this article as doi: 10.1111/ejn.13703

This article is protected by copyright. All rights reserved. 


\section{Abstract}

During conditions of increased postural instability, older adults exhibit greater lower-limb muscle cocontraction. This response has been interpreted as a compensatory postural strategy, which may be used to i006Ecrease proprioceptive information from muscle spindles or to stiffen the lower limb as a general response to minimise postural sway. The current study aimed to test these two hypotheses by investigating use of muscle co-contraction during sensory transitions that manipulated proprioceptive input. Surface EMG was recorded from the bilateral tibialis anterior and gastrocnemius medialis muscles, in young (aged 18-30) and older adults (aged 68-80) during blindfolded postural assessment. This commenced on a fixed platform (baseline: 2 mins), followed by 3minutes on a sway-referenced platform (adaptation) and a final 3-minutes on a fixed platform again (reintegration). Sensory reweighting was slower in older adults, as shown by a significantly larger and longer postural sway aftereffect once a stable platform was restored. Muscle co-contraction showed similar aftereffects, whereby older adults showed a larger increase in co-contraction once the stable platform had been restored, compared to young adults. This co-contraction aftereffect did not return to baseline until after 1 minute. Our evidence for high muscle co-contraction during the reintroduction of veridical proprioceptive input suggests that increased co-contraction in older adults is not dependent on contemporaneous proprioceptive input. Rather, it is more likely that cocontraction is a general postural strategy used to minimize postural sway, which is increased during this sensory transition. Future research should examine whether muscle co-contraction is typically a reactive or anticipatory response.

\section{Introduction}

Control of upright standing requires effective integration of sensory signals from three main sensory channels, visual, vestibular and proprioceptive, in order to form an accurate percept of the current postural system. The importance, or weight given to each channel depends on its relative reliability or accuracy and is determined using a dynamic, sensory reweighting process (Ernst \& Banks, 2002; 
Peterka, 2002). For example, when we step from a hard to a soft surface, e.g. from the pavement to sand, proprioceptive information about body sway becomes less accurate and is down-weighted, and visual and vestibular information is up-weighted to maintain postural stability. However, balance is likely to be disrupted not only when inaccurate proprioceptive information is introduced as in the example above, but also when accurate information is restored because the same sensory reweighting process is involved in both cases (Peterka \& Loughlin, 2004). Peterka and Loughlin (2004) tested this hypothesis using a sway-referencing paradigm, in which proprioceptive information from the ankle joint was rendered inaccurate by the introduction of platform rotations about the ankle joint which were an order of magnitude above the estimated excursion of the participant's centre of mass (COM). The authors found that when sway-referencing was introduced, low-frequency $(\sim 0.1 \mathrm{~Hz})$ sway was observed, reflecting underproduction of corrective torque around the ankle joint. Conversely, when a fixed surface was restored, a postural aftereffect was produced which was characterised by sway oscillations in the order of $\sim 1 \mathrm{~Hz}$ (Peterka \& Loughlin 2004), reflecting an overproduction of corrective torque around the ankle joint.

Doumas and Krampe (2010) investigated similar postural aftereffects in older adults, aged 65+. They used a paradigm in which young and older participants adapted to inaccurate proprioceptive information using sway reference for a prolonged period of time (adaptation, 18 mins) and then sway reference was removed and accurate proprioceptive information was restored (reintegration, 3 min). Older adults showed larger amounts of sway during adaptation to inaccurate proprioceptive information compared with young adults but both groups adapted to the new environment at the same rate. Similar to Peterka and Loughlin (2004), results also showed that when inaccurate proprioceptive information was introduced low frequency sway $(0-.25 \mathrm{~Hz})$ was observed, whereas the postural aftereffect witnessed when accurate information was restored was associated with high frequency sway (.5-.75 Hz). Furthermore, distinct age differences were shown between groups 
during the reintegration phase, with older adults showing significantly larger and longer postural aftereffects (as indicated by greater anterior-posterior (AP) path length). These findings were accompanied by a slower reduction of high frequency sway during the reintegration phase. This suggests that despite the lack of group differences in adaptation rates, older adults exhibited slower sensory reintegration once veridical proprioceptive information became available again, taking over twice as long as young adults to reintegrate this information to baseline levels (Doumas \& Krampe, 2010). This age-related slowing in sensory reweighting has been associated with greater postural sway and possibly falls in older adults in a number of previous studies assessing sensory integration mechanisms in postural control (Jeka et al., 2010; Eikema et al., 2012, 2013, 2014). Together, this evidence suggests that during any scenario requiring sensory readjustment, older adults may be more vulnerable to sudden perturbations due to the prolonged period of instability observed during the reweighting process.

Given older adults' heightened vulnerability to changes in sensory information, compensatory postural strategies may be required in order to support their postural stability. One such strategy is muscle co-contraction of the lower limbs (Benjuya et al., 2004), namely the simultaneous contraction of the agonist and antagonist muscles surrounding a joint, which is often equated with joint stiffening (Melzer et al., 2001; Tucker et al., 2008; Cenciarini et al., 2010). This strategy has been shown extensively in older adults during postural control tasks (Allum et al., 1998; Laughton et al., 2003; Benjuya et al., 2004; Nagai et al., 2011, 2013; Nelson-Wong et al., 2012; Craig et al., 2016). One view of muscle co-contraction proposes that it is a general postural strategy used to increase joint stiffness in an attempt to minimise postural sway (Baratta et al., 1988; Hortobágyi \& Devita, 2000; Benjuya et al., 2004; Engelhart et al., 2015). Under this view, co-contraction use would increase during any sudden increase in postural sway in both age groups, and may be generally higher in older adults compared to young adults, regardless of task difficulty, likely due to older 
adults' postural control deficits and/or smaller perceived limits of stability (Benjuya et al., 2004). However, research suggests that the use of co-contraction to reduce postural sway is a maladaptive strategy, as co-contraction has been associated with increased postural sway (Laughton et al., 2003; Nagai et al., 2011; Warnica et al., 2014) and a heightened risk of falls (Ho \& Bendrups, 2002; NelsonWong et al., 2012). This may be due to a reduction in flexibility, which can impede an adaptive response to perturbations (Tucker et al., 2008). Another view suggests that higher co-contraction in older adults may be related to a specific postural control deficit, namely an age-related reduction in proprioceptive input, and is used to enhance proprioceptive information from muscle spindles (Laughton et al., 2003; Madhavan \& Shields, 2005). If this is the case, the magnitude of cocontraction may depend on the contemporaneous quality of available proprioceptive information. Thus, manipulating the availability of accurate proprioceptive information using the paradigm used by previous studies (Doumas \& Krampe, 2010; Peterka \& Laughlin, 2004) would allow us to contrast these two views.

The main aim of the present study was to investigate whether muscle co-contraction is employed in older adults as a general postural response during periods of postural instability or a specific response related to current proprioceptive input. To this end, we manipulated the availability of accurate proprioceptive information from the ankle joint, during two sensory transitions in which we expected participants would experience significant postural sway. We assessed upright standing without vision, first on a stable surface (baseline), then on a sway-referenced surface (adaptation) and finally on a stable surface again (reintegration). We predicted that during these sensory transitions, older adults would show similar patterns of postural sway to those found by Doumas and Krampe (2010). Specifically, we predicted that sway path length would increase on initiation of sway referencing and restoration of a stable support in both age groups, but older adults would show greater and longer postural sway aftereffects once the stable surface had been restored, compared to young adults.

This article is protected by copyright. All rights reserved. 
In terms of muscle co-contraction, our predictions for baseline and adaptation were based on our previous findings (Craig et al., 2016). Specifically, at baseline, with accurate proprioceptive information available, we expected low levels of sway and co-contraction in both age groups. In contrast, during adaptation, with inaccurate proprioceptive information available and high levels of sway, we expected high levels of co-contraction, which would be significantly larger in older adults (Craig et al., 2016). Our key comparison, assessing whether co-contraction depends on the availability of accurate proprioceptive information was performed in the reintegration phase. In this phase, proprioceptive information is again accurate and postural sway increases (Peterka \& Loughlin, 2004) . If co-contraction is related to postural sway in general, then we would expect a cocontraction aftereffect, which would be greater in older adults similar to the postural sway aftereffect observed in this phase (Doumas \& Krampe, 2010). Alternatively, if co-contraction is related to afferent proprioceptive information then we would not expect to see a co-contraction aftereffect at the start of reintegration, because accurate proprioceptive information is available during this phase.

\section{Materials and methods}

\section{Participants}

Twenty-six young adults, aged 18-30, and twenty-two older adults, aged 68-80, participated in the study. Volunteers were excluded based on any previous medical history or medication use that could impair postural performance. For example, participants were immediately excluded if they gave an affirmative response to any of the following; Parkinson's disease, fall accidents within the last 6 months, hip replacement, use of orthopaedic shoes and use of tricyclic antidepressants or sleep tranquilisers. Inclusion criteria for older adults also included, scoring 25+ on the Mini-Mental State Examination (MMSE), showing typical lower limb strength/function as assessed by the Five Times Sit to Stand (5XSTS) test (Csuka \& McCarty, 1985; Bohannon, 2006), and being classified as 
independent, as determined by the Katz Basic Activities of Daily Living test (Katz et al., 1963) and the Instrumental Activities of Daily Living Scale (Lawton \& Brody, 1969). Participant withdrawal, missing motion tracking data (gaps $>500 \mathrm{~ms}$ ) and outlier analysis resulted in a final sample of twenty young adults and twenty older adults. Participant demographics (for the retained sample) can be found in Table 1. Older adults also completed the Rapid Assessment of Physical Activity (RAPA; Topolski et al., 2006). Written informed consent was obtained from all participants and the study was approved by the School of Psychology's Ethics Committee. The study conformed with the World Medical Association Declaration of Helsinki.

\section{Apparatus}

Postural assessment. Sway-referencing was induced using the Smart Balance Master (NeuroCom International, Inc., Clackamas, OR, USA). This device employs an $18^{\prime \prime} \times 18^{\prime \prime}$ dual force plate which records vertical forces at a sampling frequency of $100 \mathrm{~Hz}$ in order to derive the current medio-lateral and AP centre of pressure (COP) trajectories. The participant's CoM was approximated from these trajectories and sway-referencing was induced using a servo-controlled motor which introduced platform tilts in the sagittal plane about the ankle joint axis in proportion to the participant's expected CoM sway angle (Nashner et al., 1982). In line with Craig et al. (2016), the present study utilised the same gain levels of sway-referencing for each age group (1.0 for older adults and 1.6 for young adults) in order to eliminate age differences in postural sway. The gain levels determine the mechanical compliance of the platform. Thus, at a gain factor of 1.0 there is exact coupling between AP CoP and platform tilt in the sagittal plane. Whereas, at a gain factor of 1.6 , platform tilt is 1.6 times greater than AP CoP sway, inducing greater postural sway (Clark \& Riley, 2007), resulting in similar postural sway in young adults compared to older adults. This enabled us to examine whether older adults show higher levels of co-contraction compared to young adults even when their levels of sway are the same, similar to Craig et al. (2016). A blindfold and a safety harness, which did not restrict typical postural sway movements but prevented falling from the platform, were worn throughout postural assessment (Fig. 1).

This article is protected by copyright. All rights reserved. 
Motion capture. During postural assessment, body kinematics were assessed using a Codamotion CX1 sensor unit (Charnwood Dynamics Ltd., Rothley, Leicestershire, UK), which is an active marker system that captures motion data across three dimensions, using infrared light-emitting diodes (ILEDs). The marker set-up included: 2 platform markers, one on the fixed section of the platform and one in front of it on the posterior right corner of the moving support surface, and 4 body landmark markers (Fig. 1), which were placed at the C7 vertebra (neck level), L5 vertebra (waist level), right popliteal fossa (knee level) and right superior calcaneus (ankle level). The CX1 unit was placed behind the participant at a distance of approximately 2-metres from the ILED placed on the fixed support surface. Motion capture data were collected at a sampling rate of $100 \mathrm{~Hz}$.

EMG recordings. Surface electromyography (EMG) was used to assess co-contraction of the bilateral tibialis anterior (TA) and gastrocnemius medialis (GM) muscles during postural assessment. These muscles were chosen based on our previous paper (Craig et al., 2016) and the finding that muscle co-contraction between these two muscles has been associated with increased postural sway and falls (Nelson-Wong \& Callaghan, 2010; Warnica et al., 2014), supporting their relevance to postural stability. Disposable $\mathrm{Ag}-\mathrm{AgCl}$ electrodes (Cleartrace, CONMED, Utica, NY, USA) with an interelectrode distance of $3 \mathrm{~cm}$ were placed vertically along the muscle belly of the TA and $\mathrm{GM}$ and a ground electrode was placed on the right patella. The EMG signal was pre-amplified at a gain of 2000 using a differential amplifier (EMG100C, Biopac Systems, Inc., Santa Barbara, CA). The signal was initially band-pass filtered at $1.0-500 \mathrm{~Hz}$ and sampled at $2 \mathrm{kHz}$.

\section{Procedure}

The study was performed in a single session. In the beginning of the session, older adults completed the MMSE, RAPA and 5XSTS. The 5XSTS was performed in a chair that was $39 \mathrm{~cm}$ high and commenced with the participant's back against the chair and their arms folded across their chest. The task instructions stated, "I want you to stand up and sit down five times in a row as quickly as you can when I say 'Go'". Additionally, participants were told to extend their legs fully whilst 
standing and not to touch the back of the chair when returning to sit down. The timer was started on the 'Go' signal and stopped as soon as the buttocks touched the chair on the $5^{\text {th }}$ repetition.

The session continued for older adults, and started for young adults, with each participant performing three maximum voluntary contractions (MVCs) from each TA muscle and three reference voluntary contractions (RVCs) from each GM muscle in order to normalise the EMG recordings. Only RVCs could be reliably obtained from the GM muscles (Nelson-Wong et al., 2012). Each MVC/RVC was recorded for 2 seconds, with a 10 second rest period between each. TA MVCs were assessed during seated maximal isometric dorsiflexions of the ankle, with the knee flexed at $90^{\circ}$. GM RVCs were assessed during standing single-leg heel raises.

The postural assessment formed the final section of each session. During all blocks, participants wore a blindfold and safety harness and were instructed to stand as still as possible with their arms by their side. Participants were given two 1-min practice trials during which the platform was swayreferenced at the gain set for that age group (1.0 for older and 1.6 for young participants); the first practice trial was performed with eyes open, followed by a practice trial with eyes closed. The experimental task comprised three phases: (1) a stable 2-min baseline phase, (2) a 3-min swayreferenced adaptation phase and (3) a stable 3-min reintegration phase (Fig. 2). Participants were warned 10 seconds before the sway-referenced phase was about to commence but were not told whenever sway-referencing had stopped. EMG activity from the bilateral TA and GM muscles was recorded to assess co-contraction levels during each phase of the postural task. Motion tracking was recorded as a kinematic measure of postural sway, specifically AP path length of the waist marker was utilised as our key postural measure. This enabled the assessment of postural sway during the transitory period between platform stabilization and platform sway-referencing (and vice versa), during which the NeuroCom force plate cannot sample.

This article is protected by copyright. All rights reserved. 


\section{Data analysis}

Preliminary data pre-processing and analysis was carried out using custom-written Matlab software.

Postural measures. Gaps $(<500 \mathrm{~ms})$ in the motion tracking data from each marker were interpolated using a cubic spline routine in Matlab (Warnica et al., 2014). Following this, motion tracking data were low-pass filtered at $4 \mathrm{~Hz}$ using a $4^{\text {th }}$ order Butterworth filter. The average AP path length of the waist markers was then calculated for each 30s time window of each postural phase (Doumas \& Krampe, 2010; Craig et al., 2016).

Muscle co-contraction measures. Raw EMG data were full-wave rectified and linear envelopes were created using a $2^{\text {nd }}$ order Butterworth filter with a cut-off frequency of $4 \mathrm{~Hz}$. The experimental data from the postural trials were then normalised as a percentage of each participant's peak MVCs/RVCs (Nelson-Wong et al., 2012; Nagai et al., 2013; Warnica et al., 2014). Co-contraction indices (CCI) were calculated using the equation described in our previous paper (Lewek et al., 2004; NelsonWong et al., 2012; Craig et al., 2016). This resulted in an overall mean CCl value for each 30s window during each postural phase. Statistical analysis revealed no significant difference in $\mathrm{CCl}$ across both legs, thus the mean $\mathrm{CCl}$ values were averaged across both legs.

\section{Statistical analysis}

Statistical analyses were performed using SPSS version 23 (SPSS Statistics, IBM). Following from Doumas and Krampe's (2010) postural analysis, differences in AP path length of the waist marker and $\mathrm{CCl}$ within each phase (baseline, adaptation, reintegration) were assessed using two-way mixeddesign ANOVAs with age as between- and time window (per 30s) as within-subject factors. Differences in AP path length of the waist marker and $\mathrm{CCl}$ during the sensory transitions were assessed using mixed-design ANOVAs, which compared the mean of the baseline windows to the mean of the windows from the adaptation and reintegration phase in both age groups.

Planned contrasts and simple effects analyses were used to investigate whether; (1) we could replicate the finding that older adults show distinct postural aftereffects following sway-referencing, 
and (2) if $\mathrm{CCl}$ values follow similar patterns to postural sway during sensory transitions. Unexpected significant interactions were explored further using simple effects analyses and Bonferroni post hoc tests. Greenhouse-Geisser corrections were applied for circumstances in which sphericity could not be assumed (Mauchly's test, $p<0.05$ ).

Results

Anterior-posterior path length of the waist marker

Figure 3 provides an example of the position-time trajectories collected from the waist marker for one young and one older adult during each postural condition. From this data, the anterior-posterior path length of the waist marker was calculated as our measure of postural sway.

BASELINE. Figure 4A illustrates the mean AP path length of the waist marker results across the three postural phases in young and older adults. A mixed-design ANOVA showed no age differences in AP path length during the baseline phase. Results indicated a change in AP path length over time in both age groups, $F(3,114)=3.20, p=.03, \eta_{p}^{2}=.08$. However, Bonferroni pairwise comparisons showed no significant differences between any of the 30 s windows.

ADAPTATION. As shown in Figure 4A, the introduction of a sway-referenced support surface instilled a large increase in AP path length of the waist marker in both age groups. A mixed-design ANOVA, which compared the mean AP path length during adaptation with the mean during baseline, confirmed that AP path length was significantly higher during adaptation, $F(1,38)=224.28, p<.001$, $\eta_{p}^{2}=.86$. There was no difference between age groups or interaction between age group and phase. Analysis of AP path length throughout the 6 windows of the adaptation phase, also demonstrated no overall age differences $(p=.29)$, suggesting that our gain manipulation successfully removed any age differences in postural sway. There was also a significant decrease in path length over time shown by 
a main effect of window $F(4.03,153.23)=21.20, p<.001, \eta_{p}^{2}=.36$, and a window by age group interaction, $F(4.03,153.23)=3.87, p=.005, \eta_{p}^{2}=.09$. Simple effects analyses performed to examine this interaction showed a significant difference between age groups during window 3 only, $F(1,38)=$ 8.93, $p=.005$, in which older adults showed lower AP path length (Fig. 4A). Bonferroni pairwise comparisons showed both age groups showed significant decreases in AP path length between window $\mathrm{A} 1$ and 2 (Young: $p=.003$; Older: $p=.002$ ).

REINTEGRATION. The restoration of a stable support and veridical proprioceptive information resulted in clear aftereffects, witnessed as increased AP path length of the waist marker, especially in older adults (Fig. 4A). A mixed-design ANOVA, which compared the mean AP path length during reintegration with the mean during baseline, confirmed that AP path length was significantly higher during reintegration, $F(1,38)=63.80, p<.001, \eta_{p}^{2}=.63$. However, a significant interaction between postural phase and age group, $F(1,38)=13.33, p=.001, \eta_{p}^{2}=.26$, suggested that this increase is likely to be greater in older adults. Paired samples t-tests, with an alpha level corrected for multiple comparisons to 0.02 , indicated that both groups showed significantly higher AP path length during reintegration (Young: $t(19)=3.51, p=.002$; Older: $t(19)=7.41, p<.001$ ). Additionally, independent samples t-tests, with an alpha level corrected for multiple comparisons to 0.02 , found no age differences for either postural phase. Similarly to Doumas and Krampe (2010), the duration of this aftereffect was examined using paired samples t-tests comparing each 30 s reintegration window with the mean of the baseline windows, with an alpha level corrected for multiple comparisons to 0.008. Tests showed that for young adults the aftereffect was only significantly greater than the baseline mean in the first 30s, $t(19)=6.42, p<.001$, but in older adults the aftereffect was significant up until window 4 (2mins), $t(19)=9.59-3.44, p<.001-.003$.

This article is protected by copyright. All rights reserved. 
Analysis of AP path length of the waist marker throughout the reintegration phase, showed no overall age differences but a significant effect of time window, $F(2.40,91.11)=61.31, p<.001, \eta_{p}^{2}=$ .62. However, a significant window by age group interaction, $F(2.40,91.11)=6.22, p=.002, \eta_{p}^{2}=.14$, suggested that there may be age differences within certain windows. Simple effects analysis showed that older adults demonstrated a significantly larger aftereffect in the first reintegration window (R1), $F(1,38)=4.71, p=.04$ (Fig. 4A). Both groups showed a decline in AP path length over time (Young: $F(2.55,48.37)=18.06, p<.001$; Older: $F(1.85,35.20)=47.12, p<.001)$. Bonferroni pairwise comparisons indicated that between successive windows AP path length only declined in both groups from window R1 to R2 (Young: $p<.001$; Older: $p<.001$ ).

Muscle co-contraction (CCl)

BASELINE. Figure 4B illustrates $\mathrm{CCl}$ results across the three postural phases in young and older adults. There was no age difference and no change in $\mathrm{CCl}$ levels across time windows in the baseline phase.

ADAPTATION. As predicted from our previous study (Craig et al., 2016), exposure to a swayreferenced support surface resulted in a large increase in CCI levels (Fig. 4B). A mixed-design ANOVA, which compared the mean $\mathrm{CCl}$ levels during adaptation with the mean during baseline, confirmed that $\mathrm{CCl}$ levels were greater during adaptation, $F(1,38)=55.26, p<.001, \eta_{p}^{2}=.59$. Similarly to AP path length, there was no difference between age groups or interaction between age group and phase. Analysis of $\mathrm{CCl}$ in the 6 windows of the adaptation phase revealed that $\mathrm{CCl}$ declined over time as shown by a main effect of time window, $F(2.28,86.78)=9.99, p<.001, \eta_{p}^{2}=.21$. No overall age differences, were shown in adaptation but a window by age group interaction, $F(2.28,86.78)=2.92$, $p=.04, \eta_{p}^{2}=.07$, followed by simple effects analyses showed slightly greater $\mathrm{CCl}$ for older adults in the first window of adaptation, $F(1,38)=2.55, p=.049$ (Fig. 4B). Simple effects analyses also 
revealed that only older adults showed a main effect of time window during the adaptation phase, $F(1.92,36.48)=8.85, p=.001$. However, Bonferroni pairwise comparisons showed that only the difference between window $\mathrm{A} 1$ and $\mathrm{A} 2$ approached significance $(p=.05)$.

REINTEGRATION. Similarly to the AP path length data, an aftereffect was witnessed in CCI levels following the restoration of a stable support (Fig. 4B). Again, a mixed-design ANOVA, which compared the mean $\mathrm{CCl}$ levels during reintegration with the mean during baseline, confirmed that $\mathrm{CCl}$ levels were significantly higher during reintegration, $F(1,38)=7.53, p=.009, \eta_{p}^{2}=.17$. Similarly to the AP path length results, a phase by age group interaction, $F(1,38)=9.11, p=.005, \eta_{p}^{2}=.19$, suggested that this increase may be greater in older adults (Fig 4B). This suggestion was confirmed by paired samples t-tests with an alpha level corrected for multiple comparisons to 0.02 , showing that only older adults exhibited higher $\mathrm{CCl}$ in reintegration compared with baseline, $t(19)=4.00, p=$ .001. Like the AP path length analysis, the duration of any significant aftereffects were examined using paired samples t-tests comparing each 30 s reintegration window with the mean of the baseline windows, with an alpha level corrected for multiple comparisons to $0.008 . \mathrm{CCl}$ in reintegration was greater than in baseline in the first 30s window for young adults, $t(19)=3.06, p=$ .006 , and for 60 seconds (window R2) in older adults, $t(19)=5.42-4.35, p<.001$.

Again, no overall age differences were shown in the analysis of $\mathrm{CCl}$ levels throughout the reintegration phase but there was a main effect of time window, $F(1.73,65.63)=22.16, p<.001, \eta_{p}^{2}=$ .37. However, a time window by age group interaction, $F(1.73,65.63)=7.73, p=.002, \eta_{p}^{2}=.17$, suggested that age differences may be shown in specific windows. Simple effects analyses confirmed that older adults showed greater $\mathrm{CCl}$ than young adults up until window R3 (90 seconds), $F(1,38)=$ $6.42-2.73, p=.008-.04$. Like the AP path length data, simple effects analyses showed that both 
groups showed a significant effect of time window (Young: $F(3.07,58.39)=12.00, p<.001$; Older: $F(1.50,28.51)=15.32, p<.001)$. Bonferroni pairwise comparisons subsequently revealed that there was only a significant decline in $\mathrm{CCl}$ levels between successive windows (R1-R2) for both groups (Young: $p=.006$; Older: $p=.008$ ).

\section{Discussion}

The aim of the present study was to assess age differences in the way muscle co-contraction is used in postural control when exposed to a sensory transition. Specifically, we aimed to investigate whether greater use of muscle co-contraction in older adults may reflect a specific postural response related to incoming proprioceptive input or a more general postural response used during periods of increased postural sway. In line with our previous work, we showed that older adults displayed greater muscle co-contraction during the initiation of sway-referencing, despite showing equivalent sway to young adults during this time window (Craig et al. 2016), and they also showed larger and longer postural aftereffects when the stable surface was restored and proprioceptive information had to be reintegrated (Doumas \& Krampe, 2010). Similar aftereffects were present in muscle cocontraction in which older adults showed a larger increase in CCI levels compared to young adults, which did not return to baseline levels until after 1 minute. The presence of this co-contraction aftereffect suggests that the co-contraction response was not dependent on the availability of accurate proprioceptive information, which had been reinstated during this postural phase. Rather, muscle co-contraction may depend on current levels of postural sway, which remain high in the beginning of the reintegration phase, as witnessed in the postural sway aftereffects. This is the first study that we know of to demonstrate that muscle co-contraction shows similar aftereffects to those of postural sway measures once a stable platform has been restored.

This article is protected by copyright. All rights reserved. 
The use of muscle co-contraction in older adults

Our results concur with the previous suggestion that muscle co-contraction is utilised as a general strategy in older adults to minimise postural sway by stiffening the lower limb (Baratta et al., 1988; Hortobágyi \& Devita, 2000; Benjuya et al., 2004; Engelhart et al., 2015). An alternative view proposed that muscle co-contraction is used to enhance proprioceptive information from muscle spindles (Laughton et al., 2003; Madhavan \& Shields, 2005). However, we found that co-contraction increased both in response to inaccuracies in proprioceptive information and to the reintroduction of veridical proprioceptive information. This suggests that muscle co-contraction is unlikely to be a selective response, used to compensate a specific sensory deficit, such as reduced proprioceptive input, but instead is utilised as an over-arching postural strategy in older adults, which increases in response to postural instability, such as that induced by suboptimal sensory reweighting (as evidenced in the current study). Young adults also utilized muscle co-contraction during sensory transitions, however, older adults showed greater use of muscle co-contraction and a prolonged muscle co-contraction aftereffect, which suggests that they are more dependent on this strategy.

The idea of muscle co-contraction as an over-arching strategy in older adults was previously proposed by Benjuya and colleagues (2004). They suggested that older adults may adopt the use of this strategy whenever other strategies, such as those employed by young adults, cannot be relied upon. For example, the authors proposed that young adults can utilise proprioceptive information derived from postural sway to subsequently reduce their postural sway, whereas older adults cannot utilise this source as effectively and so rely on a non-discriminatory strategy, such as muscle cocontraction. This may be relevant to the sensory reweighting task used in the current study. For example, it may imply that as sensory reweighting is less efficient in older adults (Doumas \& Krampe, 2010; Jeka et al., 2010; Eikema et al., 2012, 2013, 2014), as supported by our current findings, older adults may utilise greater muscle co-contraction as a means to compensate for the postural instability caused by this delay.

This article is protected by copyright. All rights reserved. 
Interestingly, the $\mathrm{CCl}$ aftereffect returned to baseline in half the time that the AP path length aftereffect did in older adults. This could suggest that co-contraction helps reduce postural sway and is then relied upon less once a certain level of sway reduction has been achieved. Alternatively, it could be argued that excessive initial co-contraction may prolong the postural sway aftereffect, as antagonistic muscle actions are impeded, resulting in smaller changes in muscle length between muscle pairs and possibly obstructing perception of platform stabilization. Previous literature has suggested that muscle co-contraction is a maladaptive strategy, which has been associated with increased postural sway (Laughton et al., 2003; Nagai et al., 2011; Warnica et al., 2014) and risk of falls (Ho \& Bendrups, 2002; Nelson-Wong et al., 2012). This is likely due to the rigidity it causes in the lower limb, which can impede adaptive reactions to postural perturbations (Tucker et al., 2008).

Taken together, our results suggest that muscle co-contraction is employed as a non-discriminatory postural strategy, which may be utilised as an alternative to postural strategies that require rapid error-corrective feedback, such as sensory reweighting. This is interesting when taken in consideration with older adults' documented difficulties disengaging from a well learned pattern in task-set switching (Mayr, 2001) and the suggestion that slowed sensory reweighting may be due to inhibition problems (Redfern et al., 2009; Mendelson et al., 2010), as it suggests that older adults may show a similarly conservative response in muscle co-contraction. If muscle co-contraction is a non-discriminatory strategy, this may imply that there is a feedforward component to cocontraction, whereby older adults show a general bias towards this strategy. This could contribute to their prolonged $\mathrm{CCl}$ aftereffect, as this bias could impede older adults' ability to inhibit this response once it has been selected. This inhibition hypothesis could be tested more rigorously in future studies by using a temporal measure of muscle co-activation. The current study used an amplituderelated measure of muscle co-contraction, which examined the mean level of muscle co-contraction over a given time duration (30 seconds); however, this method cannot provide a temporal measure 
of muscle co-contraction/coactivity. Future research should examine the temporal overlap between activation of antagonistic muscles during postural assessment, similar to that which has been conducted in recent gait studies (Di Nardo et al., 2015).

\section{Study limitations and future directions}

Postural sway results in the present study largely confirmed our hypotheses, however there was one unexpected finding. We equated levels of postural sway in the two age groups during adaptation, and this was successful overall, but in the third window (60-90s) older adults showed a greater reduction in sway compared with young adults. There are two possible explanations for this result, either; older adults may show greater active engagement with postural control, due to heightened postural threat in this age group, or the higher gain for young adults could have resulted in an excessive increase in task difficultly for this group, possibly resulting in fatigue. Although the present results cannot distinguish which explanation is more plausible, the first is in line with evidence from dual-task training, which suggests that older adults adopt a "posture-first" strategy, but the opposite is the case for young adults (Doumas et al., 2009). Another limitation of the current study was the use of a single motion tracking marker as the basis of our AP path length measurement. Although it is beyond the scope of this paper, data from the waist and neck markers for this study demonstrated high cross-correlations, indicating behavior like that of an inverted pendulum, which would support use of the waist marker for this measurement. Future research should utilise additional kinematic measures in order to elucidate whether specific kinematic strategies result in differences in muscle co-contraction.

Future research could also examine the relationship between fatigue, postural sway and muscle cocontraction aftereffects. For example, one might predict that a longer exposure to sway referencing may result in a larger postural sway aftereffect due to muscle fatigue, which in turn could alter 
muscle co-contraction. Despite this, the current study found similarly sized postural sway aftereffects in older adults as those observed by Doumas and Krampe (2010), despite using a considerably smaller adaptation period ( 3 mins compared to $18 \mathrm{mins}$ ) at the same gain setting. This is consistent with the view that age differences in postural sway aftereffects are due to delayed sensory reweighting, rather than fatigue, which one would expect to be greater in the longer session. Similarly, age differences in muscle co-contraction/co-activation have been shown to be independent of muscle fatigue (Donath et al., 2015). Future assessment of postural sway and muscle co-contraction aftereffects following exposure to various sway-referencing gains could also ensure group comparisons across gains of equivalent difficulty, which could be determined by monitoring physiological factors, such as heart rate and respiration rate.

\section{Clinical implications}

The finding that co-contraction shows a similar response to the postural sway aftereffect could have important implications for research into postural control in older adults. For example, research suggests that the speed of the sensory reweighting process may depend on the level of postural threat that the current scenario imposes (Jeka et al., 2008; Polastri et al., 2012; Assländer \& Peterka, 2014; Logan et al., 2014). Under this view, the slowed sensory reweighting witnessed in the longer postural aftereffect in older adults, could reflect a conservative CNS response, whereby the CNS assigns resources to postural control based on 'functional need' (Jeka et al., 2008). Thus, due to the lack of postural threat posed by a stable platform, there is no functional need to commit these resources. However, the significant co-contraction response witnessed during the initiation of the reintegration phase suggests that the amount of instability instilled by this sensory transition still warrants CNS resources. This could support the view that the larger and longer postural aftereffects witnessed in older adults are caused by age-related deficits in sensory reweighting rather than an attempt to preserve sensory reweighting resources. Additionally, this could have significant real- 
world implications, as efficient responses to sensory transitions are a necessity for postural stability in everyday life. For example, our findings suggest that when stepping off public transport that was recently moving, it could take up to 1 minute for older adults to stop co-contracting and 2 minutes for postural sway to return to normal levels. Consequently, future research should explore differences in the utilization of sensory reweighting and muscle co-contraction in healthy older adults and those at high risk of falls, and examine whether the use of these strategies is related to perceived postural threat.

In conclusion, the combined findings from the current paper and our previous research (Craig et al., 2016) suggest that muscle co-contraction is a strategy employed more by older adults especially during sensory transitions. This study corroborates previous findings that sensory reweighting is slower in older adults (Allison et al., 2006; Doumas \& Krampe, 2010; Eikema et al., 2013) and extends these findings by reporting that $\mathrm{CCl}$ levels follow similar patterns of activity during sensory transitions. This muscle co-contraction response is likely to be a general response to postural instability, such as that caused by inefficient sensory reweighting during sensory transitions, rather than a selective response to a specific sensory deficit, such as deficient proprioceptive input. This interpretation is in line with previous suggestions that muscle co-contraction reflects a stiffening response to reduce the degrees of freedom of the body and deviations in the CoP. Furthermore, our finding that this response continues despite the restoration of a stable environment, suggests that the postural aftereffect witnessed in older adults induces considerable instability in this age group, which could have important implications during real-life everyday sensory transitions. Future research should examine whether the use of muscle co-contraction during sensory transitions aids or impedes reduction of body sway and whether this response is initiated by reactive or anticipatory mechanisms.

This article is protected by copyright. All rights reserved. 


\section{Acknowledgements}

This work was supported by a Department of Employment and Learning PhD studentship to C. Craig;

a British Academy/Leverhulme Small Research Grant R1067PSY to M. Doumas; and a Wellcome Trust Biomedical Vacation Scholarship to G.H.M. Calvert. The authors wish to thank Jerome Carr and Nialla Doherty for their assistance in data collection during this project.

\section{Conflict of Interest Statement}

The authors declare no conflict of interest.

\section{Author Contributions}

C.E.C. and M.D. designed the study. C.E.C. and G.H.M.C. were responsible for participant recruitment and data collection. All authors contributed to the data analysis and writing of the manuscript.

\section{Data Accessibility Statement}

The original Excel data files for each measure have been made available via Figshare, at the link: https://figshare.com/articles/CCD_co-contraction_data_xlsx/5353627.

\section{Abbreviations}

5XSTS; Five Times Sit to Stand

ADL; Katz Basic Activities of Daily Living

ANOVA; Analysis of Variance

AP; Anterior-Posterior

BMI; Body Mass Index

$\mathrm{CCl}$; Co-contraction Index

CNS; Central Nervous System

CoM; Centre of Mass

CoP; Centre of Pressure

This article is protected by copyright. All rights reserved. 
EMG; Electromyography

GM; Gastrocnemius Medialis

IADL; Instrumental Activities of Daily Living

ILEDs; Infrared Light-Emitting Diodes

MMSE; Mini Mental State Examination

MVCs; Maximum Voluntary Contractions

RAPA; Rapid Assessment of Physical Activity

RVCs; Reference Voluntary Contractions

TA; Tibialis Anterior

\section{References}

Allison, L.K., Kiemel, T., \& Jeka, J.J. (2006) Multisensory reweighting of vision and touch is intact in healthy and fall-prone older adults. Exp. Brain Res., 175, 342-352.

Allum, J.H.J., Bloem, B.R., Carpenter, M.G., Hulliger, M., \& Hadders-Algra, M. (1998) Proprioceptive control of posture: A review of new concepts. Gait Posture, 8, 214-242.

Assländer, L. \& Peterka, R.J. (2014) Sensory reweighting dynamics in human postural control. J. Neurophysiol., 111, 1852-1864.

Baratta, R., Solomonow, M., Zhou, B.H., Letson, D., Chuinard, R., \& D'Ambrosia, R. (1988) Muscular coactivation. The role of the antagonist musculature in maintaining knee stability. Am. J. Sports Med., 16, 113-122.

Benjuya, N., Melzer, I., \& Kaplanski, J. (2004) Aging-induced shifts from a reliance on sensory input to muscle cocontraction during balanced standing. journals Gerontol. A, Biol. Sci. Med. Sci., 59, 166-171.

Bohannon, R.W. (2006) Reference values for the five-repetition sit-to-stand test: a descriptive metaanalysis of data from elders. Percept. Mot. Skills, 103, 215-222.

Cenciarini, M., Loughlin, P.J., Sparto, P.J., \& Redfern, M.S. (2010) Stiffness and damping in postural control increase with age. IEEE Trans. Biomed. Eng., 57, 267-275.

Clark, S. \& Riley, M.A. (2007) Multisensory information for postural control: sway-referencing gain shapes center of pressure variability and temporal dynamics. Exp. brain Res., 176, 299-310.

Craig, C.E., Goble, D.J., \& Doumas, M. (2016) Proprioceptive acuity predicts muscle co-contraction of the tibialis anterior and gastrocnemius medialis in older adults' dynamic postural control. Neuroscience, 322, 251-261.

Csuka, M. \& McCarty, D.J. (1985) Simple method for measurement of lower extremity muscle strength. Am. J. Med., 78, 77-81.

Di Nardo, F., Mengarelli, A., Maranesi, E., Burattini, L., \& Fioretti, S. (2015) Assessment of the ankle muscle co-contraction during normal gait: A surface electromyography study. J. Electromyogr. Kinesiol., 25, 347-354.

This article is protected by copyright. All rights reserved. 
Donath, L., Kurz, E., Roth, R., Zahner, L., \& Faude, O. (2015) Different ankle muscle coordination patterns and co-activation during quiet stance between young adults and seniors do not change after a bout of high intensity training. BMC Geriatr., 15, 19.

Doumas, M. \& Krampe, R.T. (2010) Adaptation and reintegration of proprioceptive information in young and older adults' postural control. J. Neurophysiol., 104, 1969-1977.

Doumas, M., Rapp, M.A., \& Krampe, R.T. (2009) Working memory and postural control: adult age differences in potential for improvement, task priority, and dual tasking. Journals Gerontol. Ser. B Psychol. Sci. Soc. Sci., 64, 193-201.

Eikema, D.J.A., Hatzitaki, V., Konstantakos, V., \& Papaxanthis, C. (2013) Elderly adults delay proprioceptive reweighting during the anticipation of collision avoidance when standing. Neuroscience, 234, 22-30.

Eikema, D.J.A., Hatzitaki, V., Tzovaras, D., \& Papaxanthis, C. (2012) Age-dependent modulation of sensory reweighting for controlling posture in a dynamic virtual environment. Age (Omaha)., 34, 1381-1392.

Eikema, D.J.A., Hatzitaki, V., Tzovaras, D., \& Papaxanthis, C. (2014) Application of intermittent galvanic vestibular stimulation reveals age-related constraints in the multisensory reweighting of posture. Neurosci. Lett., 561, 112-117.

Engelhart, D., Pasma, J.H., H, Schouten, A.C., Aarts, R.G.K.M., Meskers, C.G.M., Maier, A.B., \& van der Kooij, H. (2015) Adaptation of multi-joint coordination during standing balance in healthy young and healthy old individuals. J. Neurophysiol., 216, jn.00030.2015.

Ernst, M.O. \& Banks, M.S. (2002) Humans integrate visual and haptic information in a statistically optimal fashion. Nature, 415, 429-433.

Ho, C.Y. \& Bendrups, A.P. (2002) Ankle reflex stiffness during unperceived perturbation of standing in elderly subjects. J. Gerontol. A. Biol. Sci. Med. Sci., 57, B344-B350.

Hortobágyi, T. \& Devita, P. (2000) Muscle pre- and coactivity during downward stepping are associated with leg stiffness in aging. J. Electromyogr. Kinesiol., 10, 117-126.

Jeka, J.J., Allison, L.K., \& Kiemel, T. (2010) The dynamics of visual reweighting in healthy and fallprone older adults. J. Mot. Behav., 42, 37-41.

Jeka, J.J., Oie, K.S., \& Kiemel, T. (2008) Asymmetric adaptation with functional advantage in human sensorimotor control. Exp. Brain Res., 191, 453-463.

Katz, S., Ford, A.B., Moskowitz, R.W., Jackson, B.A., \& Jaffe, M.W. (1963) Studies of illness in the aged. The index of ADL: A standardised measure of biological and psychological function. JAMA, 185, 914-919.

Kurlowicz, L. \& Wallace, M. (1999) The Mini Mental State Examination (MMSE). Director, 7, 62.

Laughton, C.A., Slavin, M., Katdare, K., Nolan, L., Bean, J.F., Kerrigan, D.C., Phillips, E., Lipsitz, L.A., \& Collins, J.J. (2003) Aging, muscle activity, and balance control: Physiologic changes associated with balance impairment. Gait Posture, 18, 101-108.

Lawton, M.P. \& Brody, E.M. (1969) Assessment of Older People: Self-Maintaining and Instrumental Activities of Daily Living. Gerontol. , 9, 179-186.

Lewek, M.D., Rudolph, K.S., \& Snyder-Mackler, L. (2004) Control of frontal plane knee laxity during gait in patients with medial compartment knee osteoarthritis. Osteoarthritis Cartilage, 12, 745751.

This article is protected by copyright. All rights reserved. 
Logan, D., Kiemel, T., \& Jeka, J.J. (2014) Asymmetric sensory reweighting in human upright stance. PLoS One, 9, e100418.

Madhavan, S. \& Shields, R.K. (2005) Influence of age on dynamic position sense: evidence using a sequential movement task. Exp. brain Res., 164, 18-28.

Mayr, U. (2001) Age Differences in the Selection of Mental Sets: The Role of Inhibition, Stimulus Ambiguity, and Response-Set Overlap. Psychol. Aging, 16, 96-109.

Melzer, I., Benjuya, N., \& Kaplanski, J. (2001) Age-related changes of postural control: effect of cognitive tasks. Gerontology, 47, 189-194.

Mendelson, D.N., Redfern, M.S., Nebes, R.D., \& Richard Jennings, J. (2010) Inhibitory processes relate differently to balance/reaction time dual tasks in young and older adults. Neuropsychol. Dev. Cogn. B. Aging. Neuropsychol. Cogn., 17, 1-18.

Nagai, K., Yamada, M., Mori, S., Tanaka, B., Uemura, K., Aoyama, T., Ichihashi, N., \& Tsuboyama, T. (2013) Effect of the muscle coactivation during quiet standing on dynamic postural control in older adults. Arch. Gerontol. Geriatr., 56, 129-133.

Nagai, K., Yamada, M., Uemura, K., Yamada, Y., Ichihashi, N., \& Tsuboyama, T. (2011) Differences in muscle coactivation during postural control between healthy older and young adults. Arch. Gerontol. Geriatr., 53, 338-343.

Nashner, L.M., Black, F.O., \& Wall, C. (1982) Adaptation to altered support and visual conditions during stance: patients with vestibular deficits. J. Neurosci., 2, 536-544.

Nelson-Wong, E., Appell, R., McKay, M., Nawaz, H., Roth, J., Sigler, R., Third, J., \& Walker, M. (2012) Increased fall risk is associated with elevated co-contraction about the ankle during static balance challenges in older adults. Eur. J. Appl. Physiol., 112, 1379-1389.

Nelson-Wong, E. \& Callaghan, J.P. (2010) Is muscle co-activation a predisposing factor for low back pain development during standing? A multifactorial approach for early identification of at-risk individuals. J. Electromyogr. Kinesiol., 20, 256-263.

Peterka, R.J. (2002) Sensorimotor integration in human postural control. J. Neurophysiol., 88, 10971118.

Peterka, R.J. \& Loughlin, P.J. (2004) Dynamic regulation of sensorimotor integration in human postural control. J. Neurophysiol., 91, 410-423.

Polastri, P.F., Barela, J.A., Kiemel, T., \& Jeka, J.J. (2012) Dynamics of inter-modality re-weighting during human postural control. Exp. brain Res., 223, 99-108.

Redfern, M.S., Jennings, J.R., Mendelson, D., \& Nebes, R.D. (2009) Perceptual inhibition is associated with sensory integration in standing postural control among Older adults. Journals Gerontol. Ser. B Psychol. Sci. Soc. Sci., 64, 569-576.

Topolski, T.D., LoGerfo, J., Patrick, D.L., Williams, B., Walwick, J., \& Patrick, M.B. (2006) The Rapid Assessment of Physical Activity (RAPA) among older adults. Prev. Chronic Dis., 3, A118.

Tucker, M.G., Kavanagh, J.J., Barrett, R.S., \& Morrison, S. (2008) Age-related differences in postural reaction time and coordination during voluntary sway movements. Hum. Mov. Sci., 27, 728737.

Warnica, M.J., Weaver, T.B., Prentice, S.D., \& Laing, A.C. (2014) The influence of ankle muscle activation on postural sway during quiet stance. Gait Posture, 39, 1115-1121.

This article is protected by copyright. All rights reserved. 
Figure Legends

Fig. 1. Diagram of the postural control task. The accuracy of proprioceptive information was manipulated using sway-referencing, during which the support surface tilts in proportion to body sway in the AP axis. Postural sway was assessed using infrared Codamotion markers placed at the $C 7, L 5$, right popliteal fossa, and right superior calcaneus. CCI was assessed using bilateral EMG of the TA and GM.

Fig. 2. Schematic of the postural assessment task. Postural assessment comprised 3 phases: (1) a 2min stable baseline phase, (2) a 3-minute adaptation phase, during which the support surface was sway-referenced at a gain of 1.0 for older adults and 1.6 for young adults, and (3) a final 3-minute reintegration phase, during which a stable platform was restored. During data analysis, the motion tracking and EMG data were split into 30s windows, in order to examine the temporal patterns of postural sway and muscle co-contraction within and between each postural phase. The key windows of interest were $A 1$ and R1, as these windows involved a sensory transition, in which the accuracy of proprioceptive information was altered compared to the previous window.

Fig. 3. Example raw position-time trajectories of the hip (L5) marker in the anterior-posterior direction in a young and older adult for each postural phase.

Fig. 4. (A) Mean AP path length from the waist marker of young and older adults for each 30s window of each postural phase; baseline (B1-4), adaptation (A1-6) and reintegration (R1-6) and (B) mean $\mathrm{CCl}$ values for each 30 s window of each postural phase. Error bars represent the SEM. Shaded bars during the reintegration phase represent the baseline mean and SEM. - Significant age difference $p<.05 . * / *$ Significant difference from baseline mean $p<.008$.

This article is protected by copyright. All rights reserved. 
Table 1. Sample means and standard deviations (in parentheses)

\begin{tabular}{|c|c|c|}
\hline & Young Adults & Older Adults \\
\hline Age (yrs) & $22.5(3.47)$ & $73.05(4.16)^{*}$ \\
\hline Sex (male, female) & 8,12 & 2,18 \\
\hline Height (cm) & $170.8(11.54)$ & $162.45(6.5)^{*}$ \\
\hline Weight (kg) & $67.32(11.33)$ & $62.3(12.07)$ \\
\hline BMI & $23.27(4.47)$ & $23.5(3.46)$ \\
\hline MMSE & N/A & $28.7(0.98)$ \\
\hline ADL & N/A & $8 / 8(0)$ \\
\hline IADL & N/A & $8 / 8(0)$ \\
\hline RAPA & N/A & $5.5 / 7(1.32)$ \\
\hline 5XSTS (secs) & $\mathrm{N} / \mathrm{A}$ & $11.58(2.21)$ \\
\hline
\end{tabular}

This article is protected by copyright. All rights reserved. 


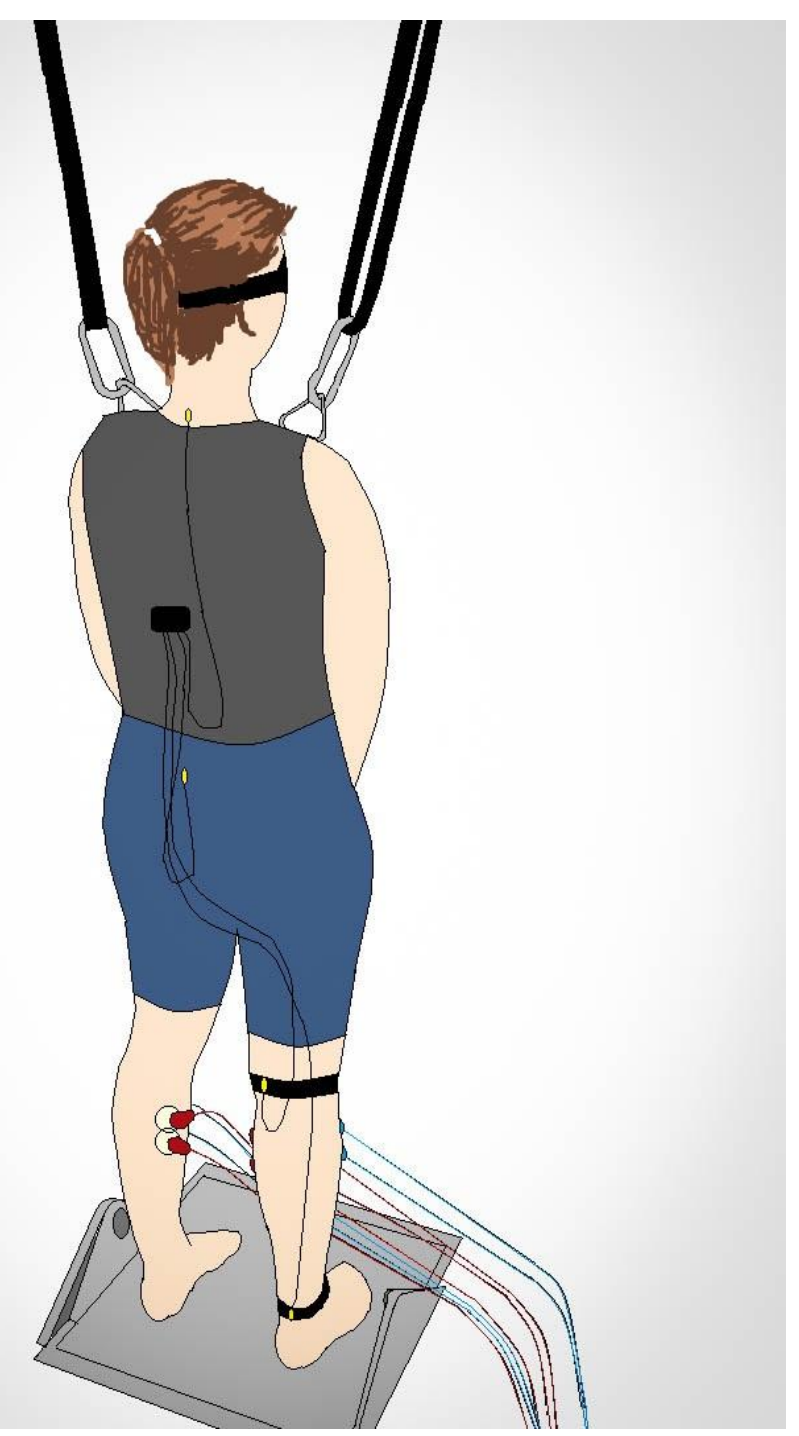

\begin{tabular}{|c|c|c|c|c|c|c|c|c|c|c|c|c|c|c|c|c|}
\hline & \multicolumn{4}{|c|}{ Stable } & \multicolumn{6}{|c|}{ Sway-referenced } & \multicolumn{6}{|c|}{ Stable } \\
\hline Phase & \multicolumn{4}{|c|}{ Baseline } & \multicolumn{6}{|c|}{ Adaptation } & \multicolumn{6}{|c|}{ Reintegration } \\
\hline Time (s) & 30 & 60 & 90 & 120 & 150 & 180 & 210 & 240 & 270 & 300 & 330 & 360 & 390 & 420 & 450 & 480 \\
\hline Window & B1 & B2 & B3 & B4 & A1 & $A 2$ & A3 & A4 & A5 & A6 & R1 & $\mathrm{R} 2$ & R3 & R4 & R5 & R6 \\
\hline
\end{tabular}

This article is protected by copyright. All rights reserved. 


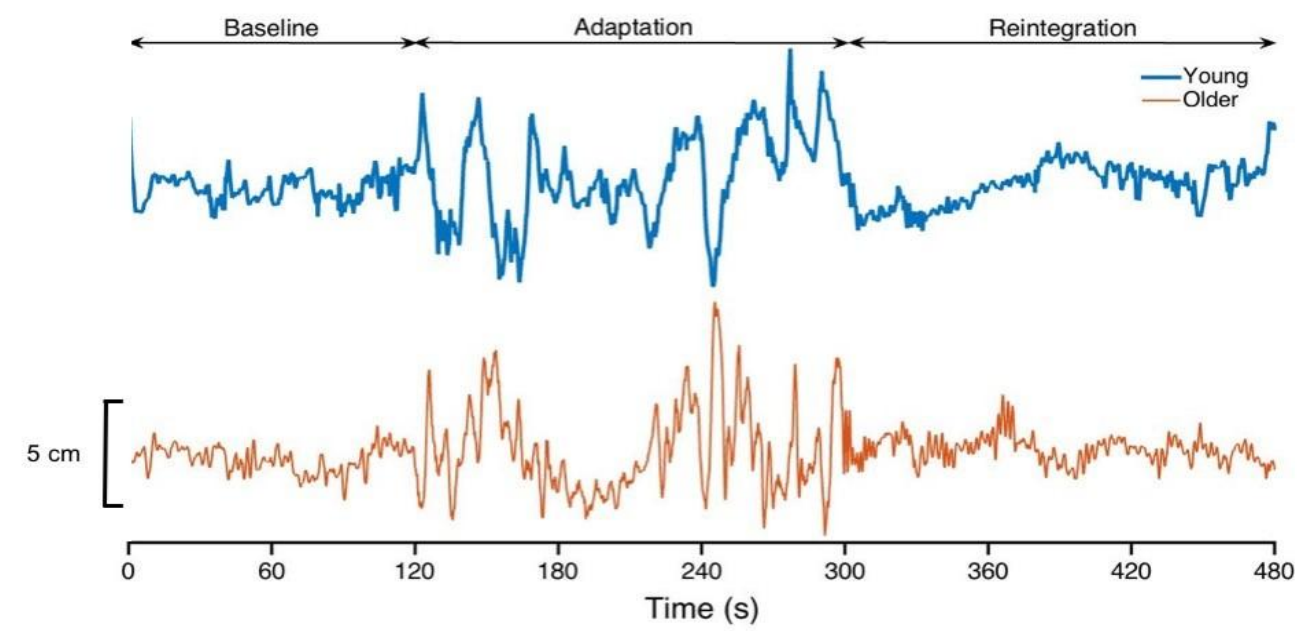

This article is protected by copyright. All rights reserved. 


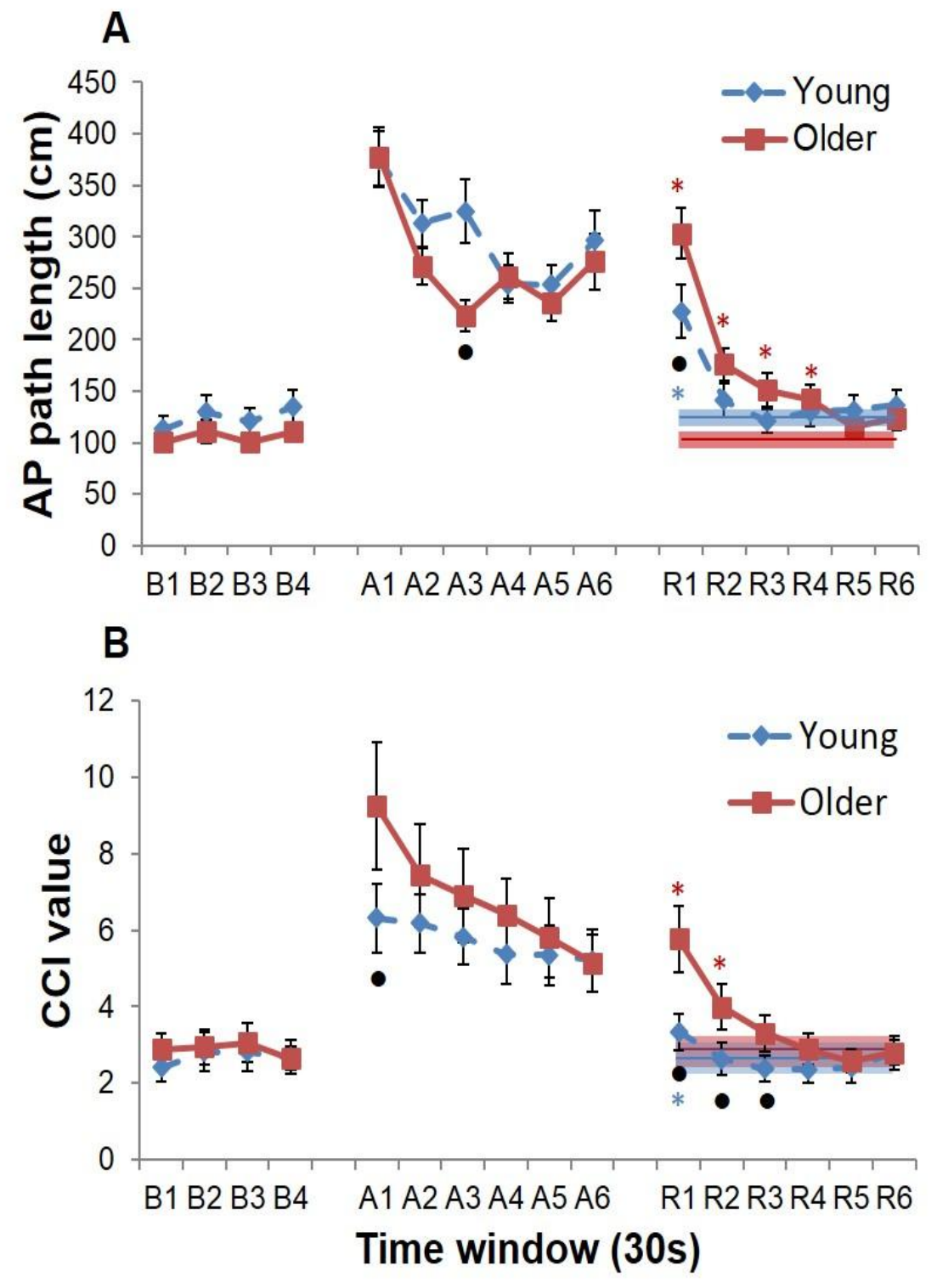

This article is protected by copyright. All rights reserved. 